University of Navarra

\title{
IMPROVING SUPPLY CHAIN EFFICIENCY THROUGH WHOLESALE PRICE RENEGOTIATION
}

\author{
Víctor Martínez de Albéniz ${ }^{1}$ \\ David Simchi-Levi ${ }^{2}$
}

${ }^{1}$ Professor, Operations Management and Technology, IESE

${ }^{2}$ Professor, Operations Research Center, MIT 


\title{
Improving Supply Chain Efficiency Through Wholesale Price Renegotiation
}

\author{
Submission: February 1, 2007 \\ Victor Martínez-de-Albéniz ${ }^{1}$ • David Simchi-Levi ${ }^{2}$
}

\begin{abstract}
In a decentralized supply chain, double marginalization is an important source of inefficiency. We suggest in this paper a simple mechanism to reduce it, that uses a wholesale price contract and renegotiation. Our mechanism only requires repeated interaction, and rational behavior from the players. Specifically, over $T$ rounds of negotiation, the supplier proposes different prices in each round, and the buyer places orders at the quoted price. Even though prices are decreasing in time, the buyer places a positive order, to force the supplier to reduce its price in the following round. This interaction results in higher profits for both supplier and buyer. We solve the buyer and supplier problems and show that, as $T$ increases, supply chain efficiency tends to $100 \%$, and the sub-optimality gap decreases with $1 / T$. Finally, we discuss how these results can be applied to design negotiation processes.
\end{abstract}

\section{Introduction}

In the past years, supply chain management has emerged as one of the most important levers for many companies to remain profitable. For example, Wal-Mart in the United States, Aldi in Germany or Mercadona in Spain have achieved remarkable profitability in retail, where margins have traditionally been very low. Key to their success are supply chain management practices, including logistics (cross-docking), demand management (every-day-low-prices) and supplier partnering (strategic suppliers).

In many industries, as the number of components outsourced has increased, managing buyersupplier relationships efficiently has become critical. One of sources of inefficiency is known as double marginalization. This phenomenon arises when two companies, working in the same supply chain, optimize inventory levels and prices taking only its own benefit into account, and thus create negative externalities for the other company. As a result, the supply chain operates in a mode that is sub-optimal in the sense that additional profit for the chain could be created and shared between its firms. Double marginalization has been studied extensively in the literature. For instance, Lariviere and Porteus [10] show that, in a supply chain with a manufacturer making to order and a retailer ordering to stock, the total stock in the supply chain is lower than the one that maximizes the supply chain profit. Indeed, the manufacturer quotes a price larger than its true cost, and, as a result, the retailer orders less than what a

\footnotetext{
${ }^{1}$ valbeniz@iese.edu (corresponding author), IESE Business School, University of Navarra, Av. Pearson 21, 08034 Barcelona, Spain

${ }^{2}$ dslevi@mit.edu, Operations Research Center, MIT, 77 Mass. Ave., Cambridge MA, 02139, USA
} 
centralized chain would. This model can be extended to more general settings, and the resulting behavior is similar.

Improvements countering double marginalization have been suggested, see Cachon [3] for an extensive review. These improvements allow the supply chain to move from local optimization, where each company takes decisions individually, considering only its own profits, towards global optimization, where the decisions of all the companies take into account aggregated supply chain profits. Typically, this is achieved by changing the incentives of the different partners in the supply chain, and aligning them towards a common goal, namely, maximizing supply chain profits. Several solutions to the problem have been proposed and implemented. Despite wide use, most of these solutions involve costly implementations. As we describe below, modifying the "natural" incentive structure of the supply chain creates additional side-effect costs.

- Buy-back agreements, see Pasternack [13], allow buyers to return unsold merchandise to the suppliers for a refund. This is extensively used in book distribution, where bookstores can return unsold items to the published for a full refund. This practice pushes bookstores to carry larger quantities of books than if they had to bear the inventory risk, i.e., the risk of discarding the excess inventory at a loss at the end of the selling season. Newspaper distributors use the same type of contract. In the case of newspapers, in order to receive a refund for unsold items, distributors usually cut the first page of each newspaper, and return it to the publisher, as proof that the item was not sold. More generally, reverse logistics must be put in place, and can be expensive for bulky or heavy items, such as books.

- Revenue sharing agreements, see Cachon and Lariviere [4], induce the same incentive effect. This type of contract stipulates a cost per unit paid from buyer to supplier, plus a share of the buyer's revenue transferred to the supplier. It is commonly used in the video rental industry. However, for revenue sharing to be effective, the supplier must be able to monitor the sales of the buyer, of which he receives a share. Obviously, without monitoring, the buyer would rather declare a very low revenue so that the payment to the supplier is reduced. For example, in 2000, see [15], the sales monitoring company Rentrak brought Hollywood Entertainment, the second largest video store chain in the United States at the time, to court, for understating sales. The understatement would have reduced the revenue sharing payments from Hollywood to Rentrak. Hollywood ended up paying $\$ 14 \mathrm{~m}$ to Rentrak. We can thus see that, to enforce revenue sharing agreements, either partners must trust each other, or otherwise information technology investments must be installed, at high cost. 
- Finally, capacity pre-commitments, see Barnes-Schuster et al. [1] and Martínez-de-Albéniz and Simchi-Levi [12], are used in high-tech and fashion clothing manufacturing. Under such contracts, the manufacturer requires advance capacity reservation from the retailer, which is charged up-front; after accurate forecasts are obtained and final production quantities adjusted, the final order is placed by the retailer, up to the reserved capacity, for an additional execution fee. Selecting the right parameters in these contracts induces the retailer to order higher quantities, which improves supply chain efficiency.

We suggest in this paper another simple mechanism to reduce double marginalization, that uses a wholesale price contract and renegotiation. Our mechanism requires repeated interaction, and rational behavior from the players, and thus should not involve implementation costs, such as logistics or information systems. By letting the supplier modify the wholesale price a few times, we improve supply chain efficiency.

Specifically, over $T$ rounds of negotiation, the supplier proposes different prices in each round, and the buyer places orders at the quoted price. Even though prices are decreasing in time, the buyer places a positive order, to force the supplier to reduce its price in the following round. This results in higher profits for both supplier and buyer. Intuitively, this simple scheme is equivalent to using a non-linear pricing schedule, which is able to reduce double marginalization. In other words, the effects of renegotiation are similar to those of volume discounts, which push buyers to place larger orders by promising lower prices for the last units ordered.

Our model uses the same setting as Lariviere and Porteus [10], with multiple negotiation rounds. That is, we consider a supplier with a given production $\operatorname{cost} c$, that serves the orders placed by a buyer after the negotiation is finished. This buyer uses the total quantity ordered to serve a stochastic final demand $D$ that arrives at the end of the negotiation. The payments from buyer to supplier are done using a wholesale price contract, with a different price for each negotiation period. Thus, the buyer maximizes its expected profit given the supplier's prices, and the supplier maximizes its (deterministic) profit given the buyer's current inventory position.

Under some regularity conditions on the demand distribution, we show that the supplier and buyer's problems are well behaved, that price quotes decrease over time and that the total ordering quantity increases with the negotiation length. Hence, supply chain efficiency improves. Finally, we show that as the number of negotiation periods goes to infinity, the supply chain profit converges to the first-best, i.e., the profit of the centralized supply chain. In addition, the sub-optimality gap decreases with $1 / T$. With these results in hand, we discuss 
in the conclusion with a very simple model how the negotiation process should be organized.

The paper contains two main contributions to the literature. First, we provide the supply chain literature with a scheme to effectively improve supply chain efficiency, as an alternative to supply contract engineering. The logic of our scheme is quite different from the existing coordinating mechanisms: it is dynamic instead of designed in one shot, statically; it is decentralized, with both players taking decisions unilaterally without considering the optimal supply chain, instead of relying on a comparison to the centralized supply chain; and finally, the improvement is progressive and efficiency increases with the length of the negotiation.

Second, our model extends previous work from the economics literature on price skimming, in the case where the buyer is strategic, in a context of supply chain. Strategic customers have been studied before, but this paper considers the market power of buyers as well. That is, in our model, the buyer takes into account the impact of its purchasing decisions on future prices, in contrast with the literature, e.g., Besanko and Winston [2]. In addition, our model can be used for further extensions with many buyers and many suppliers, where buyers are not only strategic but can use their market power.

We start by discussing the literature relevant to this work in Section 2, and turn to the model in Section 3 and an example in Section 4. We present our results in Section 5 and analyze supply chain efficiency improvements in Section 6. We conclude the paper in Section 7 with a summary of the insights and further research. All the proofs are contained in the appendix.

\section{Literature Review}

This paper directly extends Lariviere and Porteus [10], where the inefficiencies of double marginalization are analyzed. Perakis and Roels [14] investigate how serious double marginalization can be. For this purpose, they study the worst-case performance of supply chains, among all possible demand distributions, by considering the price of anarchy, i.e., the worstcase ratio between profits achieved by a decentralized supply chain and a centralized one. They show that, for the supply chain configuration used in this paper (simple push supply chain with 2 stages), as the cost-to-price ratio decreases, supply chain efficiency worsens, and approaches zero. The model of Lariviere and Porteus [10] has also been used to examine actions to reduce double marginalization. Debo and Sun [5] consider a repeated game and investigate when supply chain collaboration can be sustained. They find that, when the discount rate for future profits is high, it is more difficult to achieve supply chain collaboration.

Improving supply chain efficiency is the purpose of the supply contracts literature, which 
focuses on aligning supply chain incentives. Cachon [3] provides an excellent review of the field. Pasternack [13], Cachon and Lariviere [4], Barnes-Schuster et al. [1], Eppen and Iyer [7], among others, present supply contracts that move the supply chain towards better coordination.

Some papers from the revenue management literature are also related to ours, as we study the pricing problem of the supplier. Talluri and van Ryzin [16] provide an overview of the literature, and devote one section to price skimming models. Elmaghraby and Keskinocak [6] provide an interesting review of the literature: our work falls into their replenishment/strategiccustomers category, since we have no capacity constraint, and the buyer considers its effect on the supplier's pricing strategy. Lazear [11] develops a model where demand is constant, equal to one unit, but the buyer's valuation is uncertain and uniformly distributed. The buyer is myopic, in the sense that he places an order as soon as the price is below its valuation. The price schedule can thus be described up-front, and decreases over time, so that, in expectation, more revenue can be extracted from the buyer. Granot et al. [9] extend Lazear's model by introducing competition between suppliers. Closer to our work is the model of Besanko and Winston [2], that consider one supplier and many buyers. They introduce the notion of strategic customers, i.e., when the buyers anticipate price decreases before placing their orders. They implicitly assume that the buyers have no market power, i.e., their strategy has no impact on the supplier's price. In contrast, since we consider a single buyer, we take into account how the buyer's ordering strategy influences the supplier's prices. Finally, Erhun et al. [8] describe a similar model to ours, where one supplier faces one buyer, with the difference that in their model the demand is deterministic and linear with price. Interestingly, they observe, as we do, that supply chain efficiency is improved as the negotiation is extended.

\section{The Model}

We consider a firm, that we call the newsvendor or the buyer, that has a single opportunity to serve a stochastic demand $D$. In order to fulfill the demand, the newsvendor must install inventory prior to the demand realization. This inventory can be ordered from a supplier. If the total order quantity is lower than the demand, then sales are lost; otherwise there is excess inventory that must be discarded for a low salvage value. We denote by $f$ the p.d.f. of the demand, and by $F$ its c.d.f. $F$. Let $\bar{F}=1-F$. Without loss of generality, let $r=1$ be the per-unit sales revenue and $v=0$ the salvage value. Thus, the expected profit of the buyer is the $r$ times the expected number of units sold, minus the purchasing cost paid to the supplier.

Upstream on the supply chain, the supplier sells to the newsvendor. Let $c \in[0,1]$ be the supplier per-unit cost. Its profit is thus the dollar sales to the buyer, minus $c$ times the total 
order quantity placed by the buyer.

The details of the interaction between supplier and buyer go as follows. There are $T$ negotiation stages. In each stage, the supplier proposes a price $p_{t}$ to the buyer, and the buyer buys $q_{t} \geq 0 . t=T$ corresponds to the first period, $t=T-1$ to the second, and so on until $t=1$ the last period where the buyer can place an order. We denote by $x_{t}$ be the cumulative order of the buyer from period $T$ up to $t+1$, both included. Thus, we have $x_{T}=0$, and $x_{0}$ the total quantity purchased through the entire negotiation. Figure 1 summarizes the sequence of events and the notation.

\section{$x_{t}=$ cumulative quantity ordered from $T$ to $t+1$}
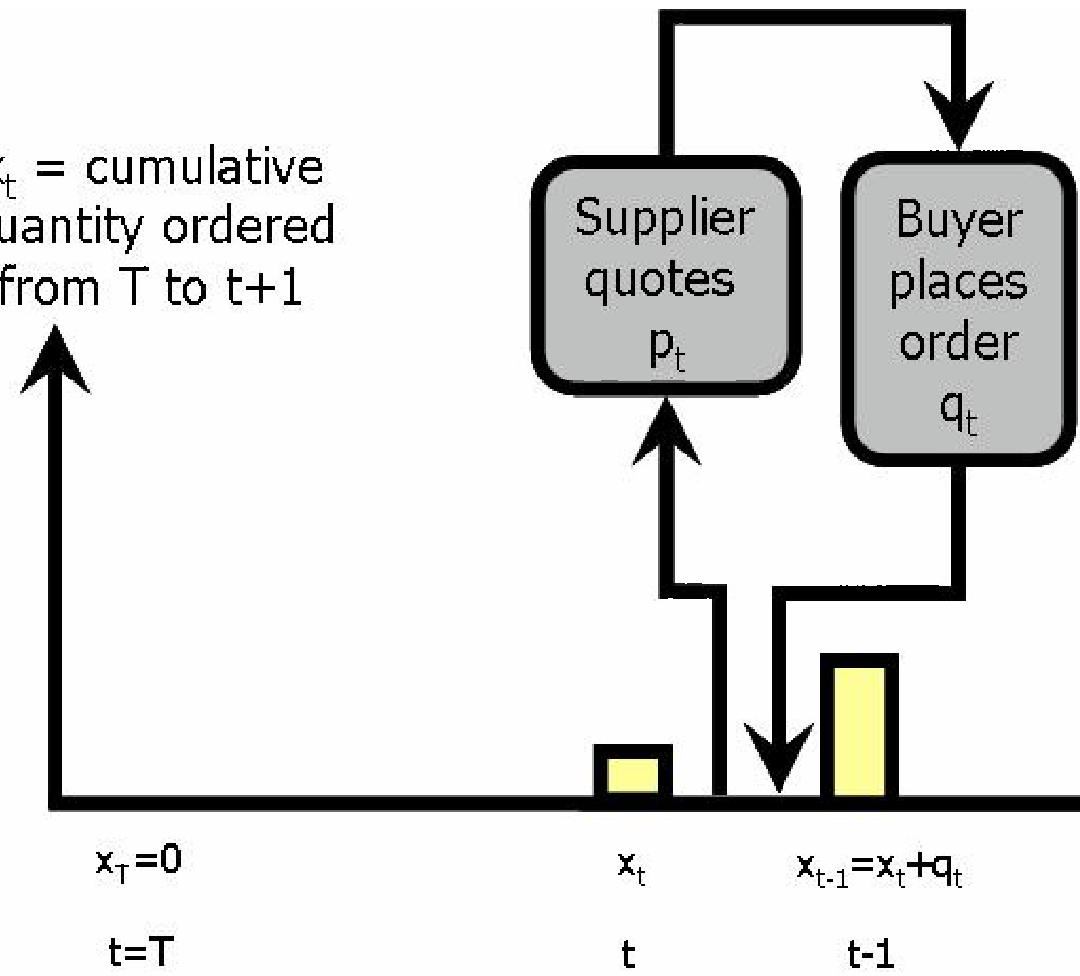

$x_{\mathrm{t}}$

$$
x_{t-1}=x_{t}+q_{t}
$$

$\mathrm{t}$ $t-1$

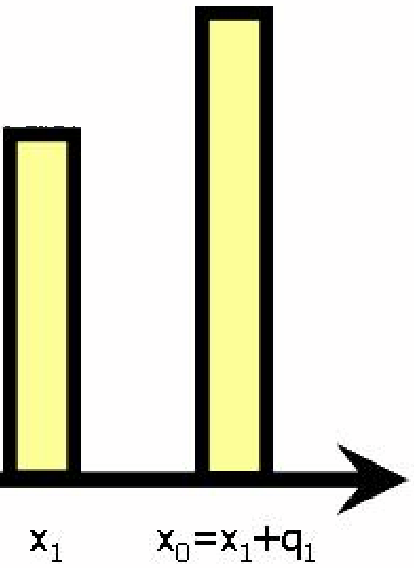

$\mathrm{t}=1 \quad \mathrm{t}=0$

Figure 1: Sequence of events.

Buyer and supplier take decisions so as to maximize their respective expected profits. We study the sub-game perfect strategies of each player. That is, for each time period $t$, and state of the world (i.e., $x_{t}$ ), the supplier sets the price $p_{t}\left(x_{t}\right)$ that maximizes its profit-to-go given the buyer's strategy; alternatively, for each $t, x_{t}$ and $p_{t}$, the buyer purchases $q_{t}\left(p_{t}, x_{t}\right)$ that maximizes its profit-to-go given the supplier's strategy. When $T=1$, our model corresponds to Lariviere and Porteus [10].

Specifically, in order to understand the players' decisions, we denote by $B_{t}\left(x_{t}\right)$ be the maxi- 
mum expected profit that the buyer can achieve with a starting stock of $x_{t}$ at time $t$, assuming that both players follow sub-game perfect strategies from $t$ to 1 . Clearly,

$$
B_{0}\left(x_{0}\right)=\mathbb{E} \min \left\{D, x_{0}\right\}=\int_{0}^{x_{0}} \bar{F} .
$$

Similarly, we denote by $S_{t}\left(x_{t}\right)$ the maximum profit that the supplier receives when the buyer has a starting stock of $x_{t}$ at time $t$. We have that $S_{0}\left(x_{0}\right)=0$, since, when the negotiation is over, the supplier cannot sell to the buyer anymore.

We can describe the buyer's problem, given $p_{t}$, as

$$
\max _{q_{t} \geq 0}\left\{-p_{t} q_{t}+B_{t-1}\left(x_{t}+q_{t}\right)\right\} \text {. }
$$

Let $q_{t}^{*}\left(p_{t}, x_{t}\right)$ be the order that maximizes the buyer's profit at time $t$. Note that when $B_{t-1}$ is concave, the optimal policy is to order up to $x_{t-1}$, where $B_{t-1}^{\prime}\left(x_{t-1}\right)=p_{t}$, i.e., $q_{t}^{*}\left(p_{t}, x_{t}\right)=$ $\max \left\{\left(B_{t-1}^{\prime}\right)^{-1}\left(p_{t}\right)-x_{t}, 0\right\}$.

Using the optimal quantity from Equation (1), the supplier's problem can simply be expressed as

$$
S_{t}\left(x_{t}\right)=\max _{p_{t}}\left\{\left(p_{t}-c\right) q_{t}^{*}\left(p_{t}, x_{t}\right)+S_{t-1}\left(x_{t}+q_{t}^{*}\left(p_{t}, x_{t}\right)\right)\right\} .
$$

From Equation (2), we obtain $p_{t}^{*}\left(x_{t}\right)$ and the corresponding $x_{t-1}^{*}\left(x_{t}\right)=x_{t}+q_{t}^{*}\left(p_{t}^{*}\left(x_{t}\right), x_{t}\right)$. With this notation, we have

$$
\begin{aligned}
& S_{t}\left(x_{t}\right)=\left(p_{t}^{*}\left(x_{t}\right)-c\right)\left(x_{t-1}^{*}\left(x_{t}\right)-x_{t}\right)+S_{t-1}\left(x_{t-1}^{*}\left(x_{t}\right)\right) \\
& B_{t}\left(x_{t}\right)=-p_{t}^{*}\left(x_{t}\right)\left(x_{t-1}^{*}\left(x_{t}\right)-x_{t}\right)+B_{t-1}\left(x_{t-1}^{*}\left(x_{t}\right)\right)
\end{aligned}
$$

We observe that the problem's order and price paths depend only on the parameter $x_{t}$, the cumulative amount of orders placed before the negotiation stage $t$. In particular, the supplier implicitly fixes the buyer's order quantity by setting the right price.

Note that for each negotiation stage, $B_{t}\left(x_{t}\right)+S_{t}\left(x_{t}\right)=B_{t-1}\left(x_{t-1}\right)+S_{t-1}\left(x_{t-1}\right)-c\left(x_{t-1}-\right.$ $\left.x_{t}\right)=B_{0}\left(x_{0}\right)-c\left(x_{0}-x_{t}\right)$. Thus, the total supply chain profit only depends on the final level of orders $x_{0}$ and the initial level of stock $x_{t}$, and not on the payments between buyer and supplier.

\section{Example and Intuition}

Consider the case of a buyer that faces a stochastic demand uniformly distributed in $[0,1]$. Consider that the production cost is $c=0$. In that case, a centralized supply chain would install inventory up to the maximum demand, i.e., $x=1$. The supply chain profits would thus be $\mathbb{E} D=0.5$. 
In the decentralized supply chain, when there is only one negotiation period, $T=1$, the supplier would set a wholesale of $w=0.5$, so that the inventory level installed by the buyer is $x=0.5$. Consequently the profit of the supplier is $w x=0.25$, while the expected profit of the buyer is $\mathbb{E} \min \{x, D\}-w x=0.125$. The total supply chain profits are thus 0.375 , only $75 \%$ of the centralized case.

Consider now the situation where there are two negotiation periods, $T=2$, and both players take their respective optimal decisions. In the first period, $t=2$, the supplier sets a price of $w_{2}=0.5625$, so that the buyer places an order for $q_{2}=0.25$; in the second period, the supplier lowers the wholesale price to $w_{1}=0.375$, and the buyer places an additional order for $q_{1}=0.375$. Thus the total inventory purchased is $x=0.625$, which yields profits of $w_{2} q_{2}+w_{1} q_{1}=0.28125>$ 0.25 for the supplier and $\mathbb{E} \min \{x, D\}-w_{2} q_{2}-w_{1} q_{1}=0.1484375>0.125$. Thus, both supplier and buyer win.

One may wonder why the buyer places an order at price $w_{2}>0.5$. Indeed, the buyer can perfectly anticipate the decrease in price at the next period, $t=1$. However, its rational choice is to purchase $q_{2}>0$ : by placing a positive-quantity order, it takes into account that this will result in a price decrease even larger than if no order was placed. This improves its overall profits.

Through the example above, we can see how extending the negotiation length $T$ can benefit both players. In the next section we develop conditions under which the buyer and supplier problems are well-behaved, and characterize the optimal supplier pricing and buyer purchasing strategies.

\section{The $T$-periods Negotiation}

Clearly, the optimality problems in Equations (1) and (2) have interior unique solutions if and only if:

- for all $t$ and $p_{t}, B_{t}(z)-p_{t} z$ is pseudo-concave in $z$ : in that case, $p_{t}^{*}\left(x_{t}\right)=B_{t-1}^{\prime}\left(x_{t-1}^{*}\left(x_{t}\right)\right)$;

- for all $t$ and $x_{t},\left(B_{t-1}^{\prime}(z)-c\right)\left(z-x_{t}\right)+S_{t-1}(z)$ is pseudo-concave in $z$.

It is not clear that these properties are satisfied by the recursive Equation (3), in the same way that the supplier's profit was not necessarily pseudo-concave in Lariviere and Porteus [10]. Some regularity conditions on the demand distribution are necessary for pseudo-concavity to be preserved in the recursion. 
In order to simplify the analysis, we define for each $x_{t}$, the final total order quantity $x_{0}\left(x_{t}\right)=$ $x_{0}^{*}\left(x_{1}^{*}\left(\ldots x_{t-1}^{*}\left(x_{t}\right) \ldots\right)\right)$. Let $\phi_{t}$ such that

$$
\phi_{t}\left(x_{0}\right)=x_{0}-x_{t}
$$

$\phi_{t}$ relates $x_{t}$, the cumulative order placed from $T$ up to $t+1$, to the total order placed from $T$ to 1 , assuming that supplier and buyer follow their optimal strategies from $t$ to 1 . Hence, we have that $x_{t}=\left(i d-\phi_{t}\right)\left(x_{0}\right)$. In addition, it is clear that $\phi_{0} \equiv 0$.

It turns out that we can rewrite in relatively simple way $B_{t}$ and $S_{t}$ as functions of $x_{0}$. Let $b_{t}\left(x_{0}\right)=B_{t}\left(\left(i d-\phi_{t}\right)\left(x_{0}\right)\right)$ and $s_{t}\left(x_{0}\right)=S_{t}\left(\left(i d-\phi_{t}\right)\left(x_{0}\right)\right)$. Working with $x_{0}$ instead of $x_{t-1}$, we can rewrite the buyer's problem of Equation (1) as

$$
\max _{x_{0} \geq x_{t}}\left\{-p_{t}\left(x_{0}-\phi_{t-1}\left(x_{0}\right)-x_{t}\right)+b_{t-1}\left(x_{0}\right)\right\} .
$$

For the maximization problem to have a unique interior solution, we must have that $-p_{t}+$ $p_{t} \phi_{t-1}^{\prime}\left(x_{0}\right)+b_{t-1}^{\prime}\left(x_{0}\right)=0$ has a unique solution, and is positive before, and negative after that solution. It is thus sufficient that $\frac{b_{t-1}^{\prime}\left(x_{0}\right)}{1-\phi_{t-1}^{\prime}\left(x_{0}\right)}$ is decreasing for all $x_{0}$.

In that case, for each $p_{t}$, the buyer selects a unique $x_{0}^{*}$ such that $u_{t}\left(x_{0}\right)=p_{t}$, where

$$
u_{t}\left(x_{0}\right):=\frac{b_{t-1}^{\prime}\left(x_{0}\right)}{1-\phi_{t-1}^{\prime}\left(x_{0}\right)} .
$$

In particular, $u_{1}\left(x_{0}\right)=\bar{F}\left(x_{0}\right)$.

Using this observation in Equation (2) allows us to rewrite the equation into

$$
s_{t}\left(x_{t}\right)=\max _{x_{0} \geq x_{t}}\left\{\left(u_{t}\left(x_{0}\right)-c\right)\left(x_{0}-\phi_{t-1}\left(x_{0}\right)-x_{t}\right)+s_{t-1}\left(x_{0}\right)\right\} .
$$

The theorem below provides the conditions to ensure that both the buyer's and the supplier's problem have a unique optimal solution, and that both $\phi_{t}$ and $u_{t}$ are well-defined.

Theorem $1 \phi_{0} \equiv 0$ and $u_{1} \equiv \bar{F}$, and for all $t \geq 1$, when $u_{t}(x)$ and $\phi_{t}(x)-x$ are decreasing for $x<\bar{F}^{-1}(c)$,

$$
\phi_{t}(x)=\phi_{t-1}(x)+\frac{\bar{F}(x)-c}{-u_{t}^{\prime}(x)}
$$

and

$$
u_{t+1}(x)=u_{t}(x)+\frac{\bar{F}(x)-c}{1-\phi_{t}^{\prime}(x)} .
$$

If the recursion is well defined, i.e., for all $k \leq t, \phi_{k}(x)-x$ and $u_{k}(x)$ are decreasing for $x<\bar{F}^{-1}(c)$, then

- $\phi_{k} \geq \phi_{k-1}$ and $u_{k} \geq u_{k-1}$; 
- and we can express the buyer's and supplier's profit as

$$
\begin{gathered}
b_{t}\left(x_{0}\right)=\int_{0}^{x_{0}} \bar{F}-\sum_{k=1}^{t} u_{k}\left(x_{0}\right)\left(\phi_{k}\left(x_{0}\right)-\phi_{k-1}\left(x_{0}\right)\right), \\
s_{t}\left(x_{0}\right)=\sum_{k=1}^{t}\left(u_{k}\left(x_{0}\right)-c\right)\left(\phi_{k}\left(x_{0}\right)-\phi_{k-1}\left(x_{0}\right)\right),
\end{gathered}
$$

where $x_{0}$ satisfies $\phi_{t}\left(x_{0}\right)=x_{0}-x_{t}$.

The theorem characterizes recursively $\phi_{t}$, that allows us to retrieve the optimal control from the supplier's point of view, and $u_{t}$, that determines the buyer's response to the supplier's price. In addition, it relates these to the supplier's and buyer's objective function.

As pointed out above, for the recursion to be well defined, we need the demand distribution, through $\bar{F}$, to satisfy some regularity conditions. As $t>1$, it becomes increasingly difficult to verify that $\phi_{t}(x)-x$ and $u_{t}(x)$ are decreasing. Thus, we develop the following results, that lead to these regularity conditions.

Theorem 2 Consider $\phi_{t}, u_{t}$ satisfying Equations (8) and (9).

Let $\lambda_{t}(p)=\phi_{t}\left(\bar{F}^{-1}(p+c)\right), v_{t}(p)=u_{t}\left(\bar{F}^{-1}(p+c)\right)$ and $g(p)=f\left(\bar{F}^{-1}(p+c)\right)$.

Then $\lambda_{t}$ and $v_{t}$ satisfy $\lambda_{0} \equiv 0, v_{1}(p)=p+c$, and for all $t \geq 1$,

$$
\lambda_{t}(p)=\lambda_{t-1}(p)+\frac{p}{g(p) v_{t}^{\prime}(p)}
$$

and

$$
v_{t+1}(p)=v_{t}(p)+\frac{p}{1+g(p) \lambda_{t}^{\prime}(p)} .
$$

In addition, for all $t \geq 0, \phi_{t}(x)-x$ and $u_{t+1}(x)$ are decreasing for $x<\bar{F}^{-1}(c)$ if and only if $\lambda_{t}(p)-\bar{F}^{-1}(p+c)$ and $v_{t+1}(p)$ are increasing for $p>0$.

This reformulation simplifies the analysis. Indeed, both the cost and the demand distribution have been collapsed into a single parameter, the function $g(p)=f\left(\bar{F}^{-1}(p+c)\right)$. To use Theorem $1, g$ must have some regularity properties. Interestingly, this function is related to the log-concavity of the demand distribution. Indeed, $g$ is concave if and only if $f^{\prime} / f$ is non-increasing, i.e., $f$ is log-concave, since

$$
g^{\prime}(\bar{F}(x)-c)=-\frac{f^{\prime}(x)}{f(x)} .
$$

Note that the demand distribution is log-concave for uniform, exponential, gamma or normal demands, among many others. Next, we solve the recursion of Equations (10) and (11) for selected demand distributions. 


\subsection{Uniform demand}

Lemma 1 Consider $g(p)=a p^{b}$, with $b \leq 2$. Then the solution to the recursive equations (10) and (11) is given by $\lambda_{t}=\bar{\lambda}_{t} p^{1-b}$ and $v_{t+1}=\bar{v}_{t+1} p+c$, where, for all $t \geq 0$,

$$
\bar{\lambda}_{t}=\frac{1}{a(1-b)}\left\{\frac{\prod_{k=1}^{t}(2-b) k}{\prod_{k=1}^{t-1}(2-b) k+1}-1\right\}
$$

and

$$
\bar{v}_{t+1}=\frac{\prod_{k=1}^{t}(2-b) k+1}{\prod_{k=1}^{t}(2-b) k}
$$

Notice that the case with $b=0$ corresponds to the case of the uniform distribution. The case $b=1+1 / \beta$, with $\beta>1$ corresponds to a Pareto distribution with finite mean, i.e., $\bar{F}(q)=(1+q)^{-\beta}$, with $c=0$. The case $b=1$ corresponds to the exponential distribution with $c=0$. Note that when $b=1, \bar{v}_{t}=t$ and $\bar{\lambda}_{t}=\frac{1}{a}\left(1+\frac{1}{2}+\ldots+\frac{1}{t}\right)$.

In addition, Lemma 1 can be used to establish the properties around 0 of the solutions to Equations (10) and (11) for any demand distribution.

Lemma 2 Let $\lambda_{t}$ and $v_{t}$ be the solutions of Equations (10) and (11), with $g$ such that $g(0)>0$. Then, for all $t \geq 0$,

$$
\lambda_{t}(0)=0, \frac{d \lambda_{t}}{d p}(0)=\frac{1}{g(0)}\left(\frac{2^{2 t}(t !)^{2}}{(2 t) !}-1\right)
$$

and

$$
v_{t+1}(0)=c, \frac{d v_{t+1}}{d p}(0)=\frac{(2 t+1) !}{2^{2 t}(t !)^{2}} .
$$

Lemma 2 characterizes the slope of the function $\lambda_{t}$ around 0 . This result, as we see in Section 6 , allows us to derive the asymptotic efficiency of the supply chain for large $t$.

\subsection{Approximation of normal demand}

Lemma 3 Consider $g(p)=a p\left(1-\frac{p}{r}\right)$, with $a \geq 0, r \geq 1-c$. Then the solution to the recursive equations (10) and (11) is given by

$$
\lambda_{t}=\left(\frac{1}{a}\right)\left\{\sum_{k=1}^{t} \frac{1}{k}\left(1-\frac{p}{r}\right)^{-k}\right\}
$$


and

$$
v_{t+1}=c+r\left\{1-\left(1-\frac{p}{r}\right)^{t+1}\right\}
$$

This lemma allows us to use a closed-form formula to approximate the normal distribution. Indeed, consider a normal distribution of average $\mu$ and standard deviation $\sigma$, and $c=0$. As shown in Figure 2, $\bar{F}$ can be approximated well by

$$
\overline{F^{a}}(x)=\frac{1}{1+e^{\frac{x-\mu}{\sigma^{a}}}} .
$$

where $\sigma^{a}=\sigma \sqrt{\frac{\Pi}{8}}$. This approximation is very accurate for values around the mean, but has heavier tails than the normal distribution.

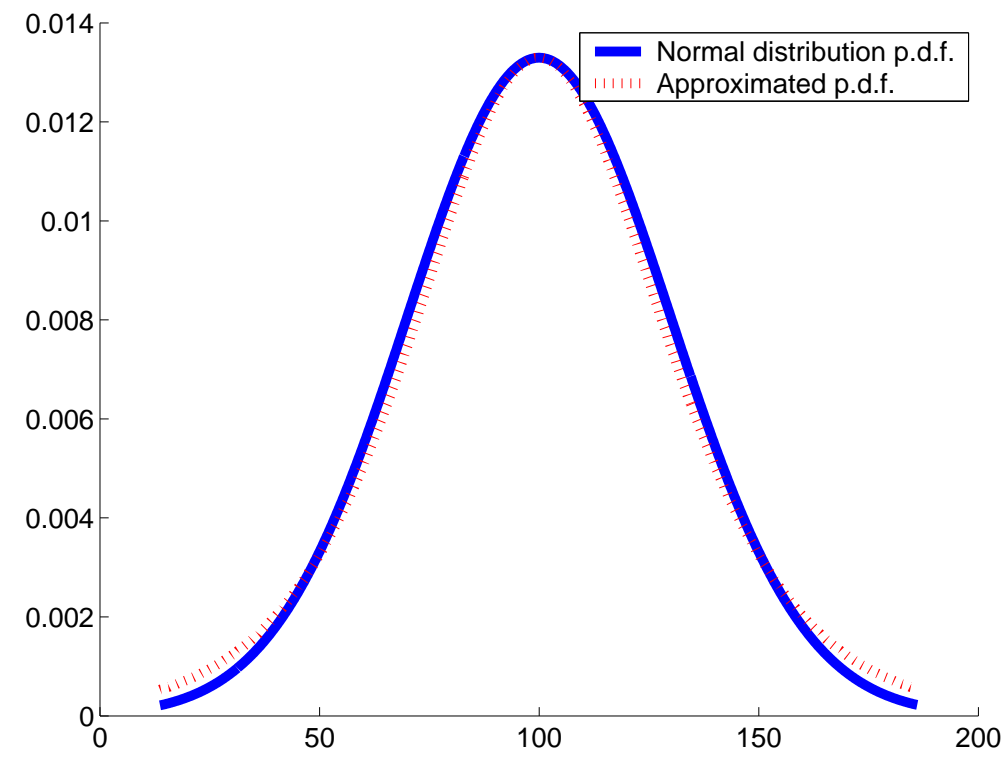

Figure 2: Comparison of the p.d.f. of the normal distribution of mean $\mu=100$ and standard deviation $\sigma=30$, with the p.d.f. $f^{a}$ with $\sigma^{a}=\sigma \sqrt{\frac{\Pi}{8}}$.

Thus, $f^{a}(x)=\frac{e^{\frac{x-\mu}{\sigma^{a}}}}{\sigma^{a}\left(1+e^{\frac{x-\mu}{\sigma^{a}}}\right)^{2}}$ and $g$ can be approximated by $g^{a}(p)=\frac{p(1-p)}{\sigma^{a}}$. Lemma 3 hence provides an approximation for the normal distribution for the specific case of $c=0$.

\subsection{Exponential demand}

For an exponential demand, we describe next the solution of the recursion presented in Theorem 2. When $\bar{F}(q)=e^{-a q}$, then $g(p)=a(p+c)$. 
Lemma 4 Consider $g(p)=p+1$. Then the solution to the recursive equations (10) and (11) is given by

$$
\lambda_{t}(p)=\frac{Q_{t}^{1}(p)}{Q_{t}^{2}(p)} \quad \text { and } \quad v_{t}(p)=c+\frac{Q_{t}^{3}(p)}{Q_{t}^{4}(p)}
$$

where $Q_{t}^{i}$ are polynomials. The sequence of polynomials satisfies the recursion

$$
\begin{aligned}
& Q_{0}^{1}=0, Q_{0}^{2}=1, Q_{1}^{3}=p, Q_{1}^{4}=1 \\
& \begin{array}{l}
Q_{t}^{2}=(p+1) Q_{t-1}^{2}\left\{\begin{array}{l}
\left.\left(Q_{t}^{3}\right)^{\prime} Q_{t}^{4}-\left(Q_{t}^{4}\right)^{\prime} Q_{t}^{3}\right\} \\
\left.\left(Q_{t}^{3}\right)^{\prime} Q_{t}^{4}-\left(Q_{t}^{4}\right)^{\prime} Q_{t}^{3}\right\}+p Q_{t-1}^{2}\left(Q_{t}^{4}\right)^{2}
\end{array}\right. \\
Q_{t}^{1}=(p+1) Q_{t-1}^{1} \\
Q_{t+1}^{4}=Q_{t}^{4}\left[\left(Q_{t}^{2}\right)^{2}+(p+1)\left\{\left(Q_{t}^{1}\right)^{\prime} Q_{t}^{2}-Q_{t}^{1}\left(Q_{t}^{2}\right)^{\prime}\right\}\right] \\
\left.Q_{t+1}^{3}=Q_{t}^{3}\left[\left(Q_{t}^{2}\right)^{2}+(p+1)\left\{Q_{t}^{1}\right)^{\prime} Q_{t}^{2}-Q_{t}^{1}\left(Q_{t}^{2}\right)^{\prime}\right\}\right]+p Q_{t}^{4}\left(Q_{t}^{2}\right)^{2} .
\end{array}
\end{aligned}
$$

For all $t \geq 1$, degree $\left(Q_{t}^{1}\right)=\operatorname{degree}\left(Q_{t}^{2}\right)$ and degree $\left(Q_{t}^{3}\right)=\operatorname{degree}\left(Q_{t}^{4}\right)+1$.

We present below the first elements of the sequence.

$$
\begin{aligned}
& Q_{1}^{1}=p, Q_{1}^{2}=p+1, \\
& Q_{2}^{3}=p(p+1)(2 p+3), Q_{2}^{4}=(p+1)(p+2) \\
& Q_{2}^{1}=p(p+1)^{3}\left(3 p^{2}+12 p+10\right), Q_{2}^{2}=2(p+1)^{5}(p+3), \\
& Q_{3}^{3}=2 p(p+1)^{9}(p+2)^{2}\left(6 p^{3}+40 p^{2}+75 p+45\right), Q_{3}^{4}=2(p+1)^{9}(p+2)^{2}\left(2 p^{3}+15 p^{2}+33 p+24\right)
\end{aligned}
$$

As $t$ grows, we obtain a sequence of polynomials with positive coefficients. In addition, we observe that these polynomials are such that $\lambda_{t}(p)=\frac{Q_{t}^{1}(p)}{Q_{t}^{2}(p)}$ and $v_{t}(p)=c+\frac{Q_{t}^{3}(p)}{Q_{t}^{4}(p)}$ are non-decreasing. These curves are illustrated in Figure 3.

Lemma 5 Consider $g(p)=a(p+b)$, with $a, b \geq 0$. Then the solution to the recursive equations (10) and (11) is given by

$$
\lambda_{t}(p)=\frac{Q_{t}^{1}\left(\frac{p}{b}\right)}{a Q_{t}^{2}\left(\frac{p}{b}\right)} \quad \text { and } \quad v_{t}(p)=c+\frac{b Q_{t}^{3}\left(\frac{p}{b}\right)}{Q_{t}^{4}\left(\frac{p}{b}\right)}
$$

This captures the case of the exponential distribution with decay rate $a: g(p)=a(p+c)$. In addition, we develop below a similar result, Lemma 6 , that allows us to use Theorem 1 for a wide class of functions. Namely, we approximate $\bar{F}$ by exponentials with increasing decay 

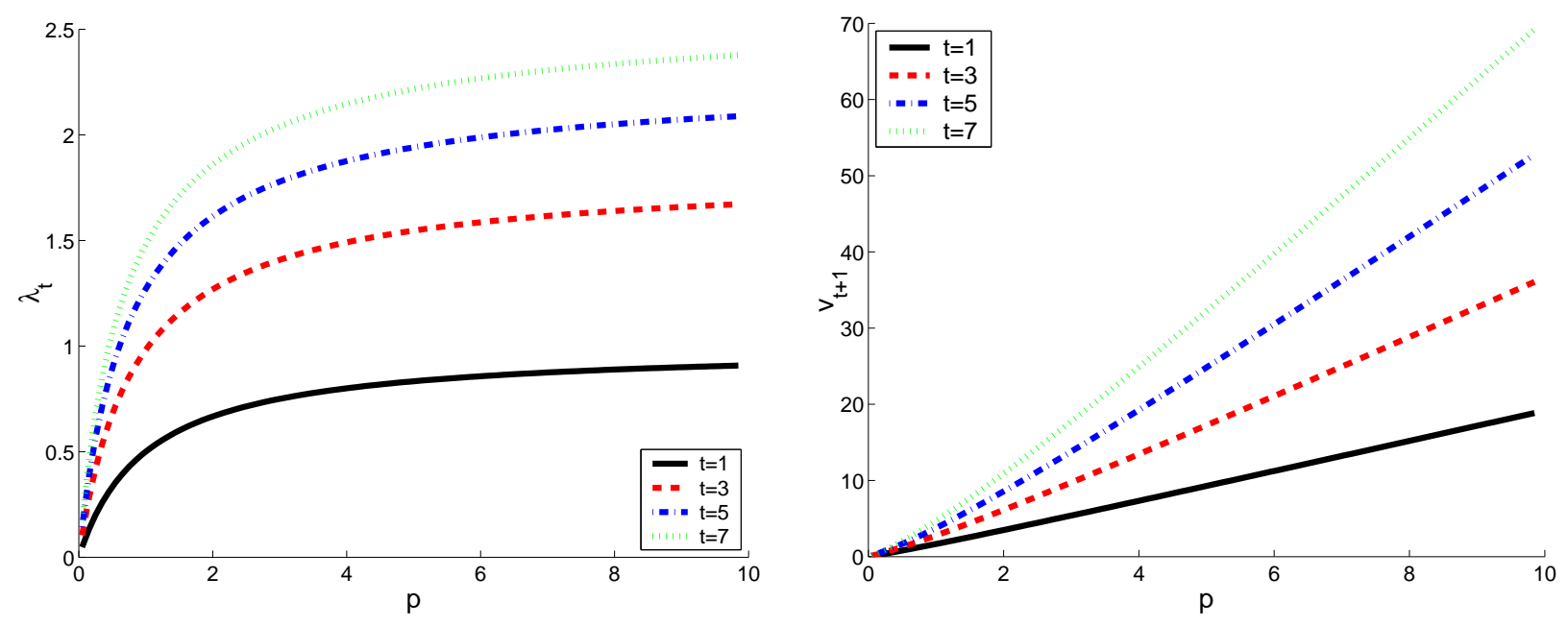

Figure 3: Plot of $\lambda_{t}$ and $v_{t+1}$ for $t=1,3,5,7$, for $g(p)=p+1$ (exponential demand). We observe that $\lambda_{t}$ is increasing concave, and $v_{t}$ increasing convex $(c=0)$.

rate: in each interval $\left[q_{i-1}, q_{i}\right]$, we have $\bar{F}(q)=f_{i} e^{-a_{i} q_{i}}$. This can be done easily for demands with increasing failure rate.

Lemma 6 Assume that the demand distribution is piecewise exponential, i.e.,

$$
f(q)=\left\{\begin{aligned}
f_{1} a_{1} e^{-a_{1} q} & \text { for } 0 \leq q<q_{1} \\
f_{2} a_{2} e^{-a_{2} q} & \text { for } q_{1} \leq q<q_{2} \\
\ldots & \\
f_{K} a_{K} e^{-a_{K} q} & \text { for } q_{K-1} \leq q<q_{K} \\
0 & \text { for } q_{K} \leq q
\end{aligned}\right.
$$

with $0 \leq a_{1} \leq \ldots \leq a_{K}$, and such that $\bar{F}\left(q_{i}\right)=f_{i} e^{-a_{i} q_{i}}$ for $i \leq K-1$.

Then for all $t$,

$$
\phi_{t}(x)=\frac{c^{2} Q_{t}^{1}\left(\frac{\bar{F}(x)}{c}-1\right)}{f(x) Q_{t}^{2}\left(\frac{\bar{F}(x)}{c}-1\right)} \quad \text { and } u_{t}(x)=c+\frac{c Q_{t}^{3}\left(\frac{\bar{F}(x)}{c}-1\right)}{Q_{t}^{4}\left(\frac{\bar{F}(x)}{c}-1\right)} \text {. }
$$

Thus, for all $t$, if $\frac{Q_{t}^{1}}{Q_{t}^{2}}$ and $\frac{Q_{t}^{3}}{Q_{t}^{4}}$ are increasing, then $\phi_{t}(x)-x$ and $u_{t}(x)$ are decreasing.

\section{Supply Chain Efficiency}

In this section, we analyze the gains of supply chain efficiency achieved by extending the length $T$ of the negotiation. For this purpose, we compare the highest supply chain expected profit, 
achieved by global optimization, to the supply chain expected profit in the decentralized setting, where buyer and supplier have $T$ negotiation periods before facing the demand.

Let $z^{*}$ be the optimal centralized quantity, that achieves global optimization: $z^{*}$ is such that $\bar{F}\left(z^{*}\right)=c$. In addition, let $S C^{*}$ be the corresponding supply chain profit. We compare $z^{*}$ and $S C^{*}$ to $z_{T}$ and $S C_{T}$, the total ordering quantity and supply chain profit, after $T$ negotiation rounds. $z_{T}$ satisfies $\phi_{T}\left(z_{T}\right)=z_{T}$.

Theorem 3 Consider $\phi_{t}$ and $u_{t}$ defined by Equations (8) and (9). Assume that, for $t \geq 0$, $\phi_{t}(x)-x$ and $u_{t+1}(x)$ are decreasing for $x<z^{*}$. Then $z_{T}$ and $S C_{T}$ are increasing in $T$. In addition,

$$
\lim _{T \rightarrow \infty} z_{T}=z^{*} \text { and } \lim _{T \rightarrow \infty} S C_{T}=S C^{*} .
$$

Thus, the efficiency of the supply chain improves with the number of negotiation rounds. In addition, the longer the time horizon, the higher the buyer and the supplier's profits, and hence the higher the supply chain profit. Both players benefit from extending the negotiation.

This result immediately leads to another question: how fast does the ordering quantity $z_{T}$ and supply chain profit $S C_{T}$ converge to the optimal $z^{*}$ and $S C^{*}$ ? It turns out that the convergence rate of the ordering quantity is independent of the demand distribution, as long some regularity conditions are satisfied, as shown below.

We consider first the uniform distribution in $\left[D_{\min }, D_{\max }\right]$. Applying Lemma 1 with $a=$ $\frac{1}{D_{\max }-D_{\min }}, b=0$, yields that for $z \in\left[D_{\min }, D_{\max }\right]$

$$
\phi_{T}(z)=\left(\frac{2^{2 T}(T !)^{2}}{(2 T) !}-1\right)\left(z^{*}-z\right)
$$

where $z^{*}=D_{\max }-\frac{c}{a}$ is the centralized optimal order quantity. The total capacity installed after $T$ negotiation stages, i.e., $z_{T}$, satisfies $\phi_{T}\left(z_{T}\right)=z_{T}$. Solving the algebra yields that

$$
\frac{z^{*}-z_{T}}{z^{*}}=\frac{(2 T) !}{2^{2 T}(T !)^{2}}
$$

The Stirling factorial approximation allows us to approximate the relative deviation to $z^{*}$ for large $T$, as

$$
\frac{z^{*}-z_{T}}{z^{*}} \approx \sqrt{\frac{1}{\Pi T}}
$$

where $\Pi \approx 3.1416$. In addition, the price trajectory proposed by the seller decreases as the negotiation advances. Using Lemma 1, we have that

$$
\frac{u_{t+1}-c}{u_{t}-c}=\frac{2 t+1}{2 t}
$$


where $u_{t}$ is the price proposed $t$ periods from the end, when there are $T$ negotiation rounds in total. The supply chain profit can be expressed as

$$
S C_{T}=\int_{0}^{z_{T}} \bar{F}(t) d t-c z_{T}
$$

while the centralized optimal profit is

$$
S C^{*}=\int_{0}^{z^{*}} \bar{F}(t) d t-c z^{*}
$$

Thus, we have

$$
S C^{*}-S C_{T}=\int_{z_{T}}^{z^{*}} \bar{F}(t) d t-\left(z^{*}-z_{T}\right) \bar{F}\left(z^{*}\right)=\int_{z_{T}}^{z^{*}}\left(t-z_{T}\right) f(t) d t .
$$

and since $f(t)=a$ and $S C^{*}=\frac{a\left(z^{*}\right)^{2}}{2}$,

$$
\frac{S C^{*}-S C_{T}}{S C^{*}}=\left(\frac{(2 T) !}{2^{2 T}(T !)^{2}}\right)^{2} .
$$

For $T=1$, the supply chain inefficiency is thus $25 \%$ and for large $T, \frac{S C^{*}-S C_{T}}{S C^{*}} \approx \frac{1}{\Pi T}$. The supply chain loss of optimality thus decreases with $1 / T$.

The split of profit between supplier and buyer can also be calculated. The supplier's profit can be expressed as

$$
\begin{aligned}
s_{T}\left(z_{T}\right) & =\sum_{k=1}^{T}\left(u_{k}\left(z_{T}\right)-c\right)\left(\phi_{k}\left(z_{T}\right)-\phi_{k-1}\left(z_{T}\right)\right) \\
& =a\left(z^{*}-z_{T}\right)^{2} \sum_{k=1}^{T} \frac{(2 k-1) !}{2^{2 k-2}((k-1) !)^{2}} \frac{2^{2 k-2}((k-1) !)^{2}}{(2 k-1) !} \\
& =a\left(z^{*}-z_{T}\right)^{2} T \\
& =2 T\left(\frac{z^{*}-z_{T}}{z^{*}}\right)^{2} \cdot S C^{*}
\end{aligned}
$$

As a result, when $T \rightarrow \infty, s_{T}\left(z_{T}\right) \rightarrow \frac{2}{\Pi} S C^{*}$. This ratio also appears in Erhun et al. [8], although with different modeling assumptions. Also, since $\frac{z^{*}-z_{1}}{z^{*}}=\frac{1}{2}, s_{1}\left(z_{1}\right)=\frac{1}{2} S C^{*}$. Thus, the maximum gain achieved by the supplier is $4 / \Pi-1 \approx 27.3 \%$. The maximum supply chain gain is $4 / 3-1=33.3 \%$, while the gain by the buyer is $(4-8 / \Pi)-1 \approx 45.6 \%$. The extension of the negotation thus benefits the buyer more than the supplier, and the supply chain share of profit for the supplier goes from $\frac{s_{1}\left(z_{1}\right)}{S C_{1}}=\frac{2}{3}=66.6 \%$ to $\frac{s_{T}\left(z_{T}\right)}{S C_{T}} \rightarrow \frac{2}{\Pi} \approx 63.7 \%$.

Interestingly, the asymptotic behavior of $z_{T}$ and $S C_{T}$ in the general case can be derived from the uniform demand case. Indeed, Lemma 2 shows that, around $z=z^{*}$ (and $p=0$ by using the 
transformation proposed in Theorem 2), the functions $\lambda_{T}$ and $\phi_{T}$ can be approximated locally by linear functions. This allows us to derive the following result.

Theorem 4 Consider $\phi_{t}$ and $u_{t}$ defined by Equations (8) and (9). Assume that $f\left(z^{*}\right)>0$ and that around $z^{*}, f$ is smooth, i.e., infinitely differentiable. Assume also that for $t \geq 0, \phi_{t}(x)-x$ and $u_{t+1}(x)$ are decreasing for $x<z^{*}$. Then, for large $T$,

$$
\frac{z^{*}-z_{T}}{z^{*}}=\frac{1}{\sqrt{\Pi}} \cdot \frac{1}{\sqrt{T}}+\epsilon^{z}\left(\frac{1}{\sqrt{T}}\right)
$$

and

$$
\frac{S C^{*}-S C_{T}}{S C^{*}}=\frac{f\left(z^{*}\right)\left(z^{*}\right)^{2}}{2 S C^{*} \Pi} \cdot \frac{1}{T}+\epsilon^{S C}\left(\frac{1}{T}\right),
$$

where $\epsilon^{i}(y) / y \rightarrow 0$ when $y \rightarrow 0$.

The theorem suggests that $z_{T}$ converges to $z^{*}$ with the square-root of $T$. In addition, $1-\frac{z_{T}}{z^{*}}$ falls with $\frac{\gamma}{\sqrt{T}}$, where $\gamma=\frac{1}{\sqrt{\Pi}}$, independent of the distribution, and relies only on the fact that the demand p.d.f. is sufficiently smooth near $z=z^{*}$. Finally, we observe that the suboptimality gap $1-\frac{S C_{T}}{S C^{*}}$ falls with $\frac{1}{T}$. The convergence coefficient does depend on the demand distribution.

Theorem 4's convergence results are illustrated by the numerical experiments below. We examine the improvement of supply chain efficiency, as a function of the length of the negotiation horizon. We focus on uniform, exponential, normal and Pareto distributions. Interestingly, Perakis and Roels [14] show that, when $T=1$, the class of Pareto distributions achieves the worst-case sub-optimality gap. As we show below, this gap is rapidly corrected as $T$ increases. Figure 4(right) shows how, for all four distributions plotted, the sub-optimality gap decreases with $\frac{1}{T}$ approximately. Figure 4 (left) shows the decrease of $1-\frac{z_{T}}{z^{*}}$. Figure 5 shows how the sub-optimality gap goes to 0 , for several distributions.

Finally, we have compared the share of the supply chain profit going to the buyer. It is relatively stable, as shown in Figure 6 . This implies that the additional profit generated by extending the negotiation horizon is shared approximately in a proportional manner, according to the initial split of profit with $T=1$.

\section{Conclusions and Discussion}

In this paper, we have presented a supply chain model where a buyer faces a stochastic demand, and must install inventory to serve this demand before it is realized. The inventory can be ordered from a supplier. Buyer and supplier interact over a multi-period horizon, where, in 

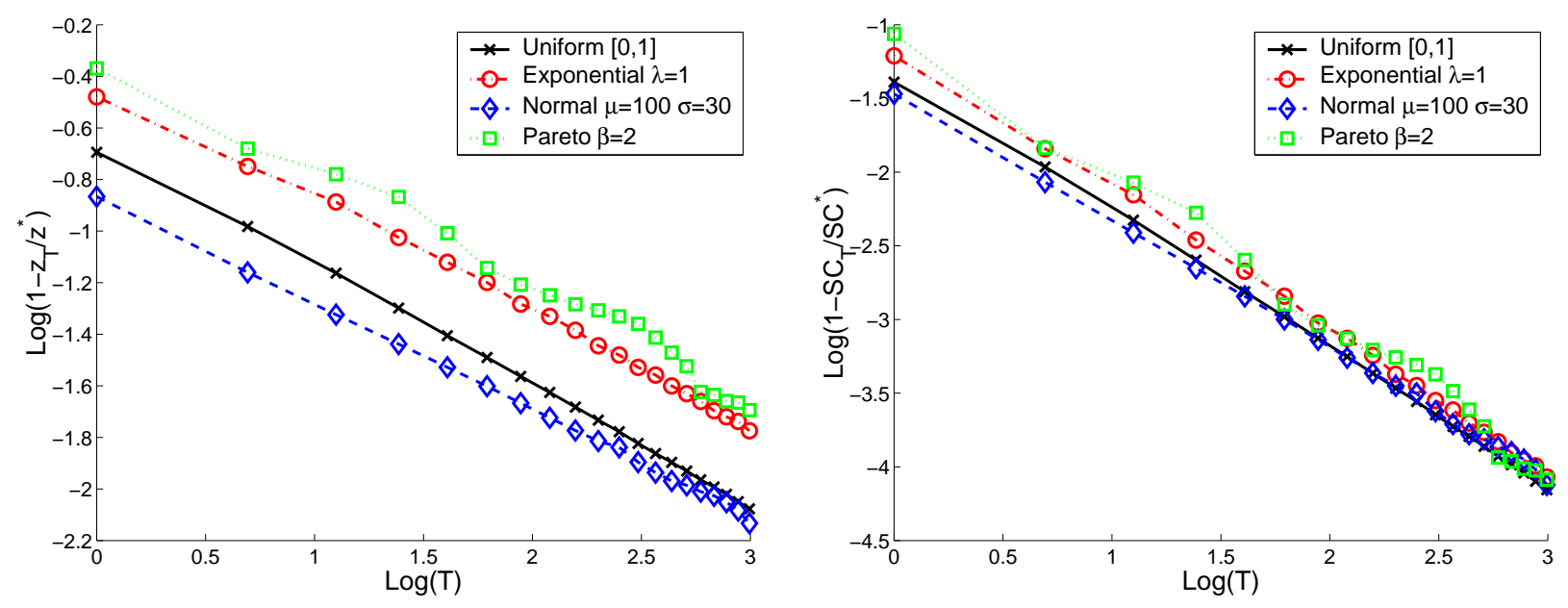

Figure 4: Evolution of $1-\frac{z_{T}}{z^{*}}$ (left) and $1-\frac{S C_{T}}{S C^{*}}$ (right) as a function of $T$, shown in a $\log -\log$ scale plot. We show the results for several demand distributions: the uniform $[0,1]$, the exponential of decay rate 1 , the normal distribution of mean 100 and standard deviation 30, and the Pareto distribution with $\bar{F}(q)=\frac{1}{(1+q)^{2}}$. We set $c=0.2$. We observe that the $\log$-log slope is approximately $-1 / 2$ for the left figure, and -1 for the right figure.

\begin{tabular}{|c|c|c|c|c|}
\hline & $T=1$ & $T=2$ & $T=5$ & $T=20$ \\
\hline Uniform $[0,1]$ & $24.9 \%$ & $14.0 \%$ & $6.0 \%$ & $1.6 \%$ \\
\hline Uniform $[5,6]$ & $8.8 \%$ & $3.3 \%$ & $1.4 \%$ & $0.4 \%$ \\
\hline Exponential $\lambda=1$ & $29.8 \%$ & $15.9 \%$ & $6.9 \%$ & $1.7 \%$ \\
\hline Normal $\mu=100, \sigma=30$ & $23.0 \%$ & $12.6 \%$ & $5.8 \%$ & $1.6 \%$ \\
\hline Normal $\mu=100, \sigma=50$ & $24.4 \%$ & $15.9 \%$ & $7.0 \%$ & $1.7 \%$ \\
\hline Pareto $\beta=2$ & $34.6 \%$ & $15.9 \%$ & $7.5 \%$ & $1.7 \%$ \\
\hline Pareto $\beta=1.1$ & $29.7 \%$ & $17.2 \%$ & $7.4 \%$ & $1.7 \%$ \\
\hline
\end{tabular}

Figure 5: Optimality gap $1-\frac{S C_{T}}{S C^{*}}$, for several demand distributions, and $c=0.2$. Note that the gap for the uniform $[5,6]$ is much smaller than the rest because there $z^{*}=5.8, S C^{*}=4.32$, and thus $\frac{f\left(z^{*}\right)\left(z^{*}\right)^{2}}{2 S C^{*}} \approx 3.89$, relatively high. This is in contrast with the uniform $[0,1]$, where $\frac{f\left(z^{*}\right)\left(z^{*}\right)^{2}}{2 S C^{*}}=1$. 


\begin{tabular}{|c|c|c|c|c|}
\hline & $T=1$ & $T=2$ & $T=5$ & $T=20$ \\
\hline Uniform $[0,1]$ & $33.4 \%$ & $34.6 \%$ & $35.6 \%$ & $36.2 \%$ \\
\hline Uniform $[5,6]$ & $0 \%$ & $2.6 \%$ & $3.5 \%$ & $4.2 \%$ \\
\hline Exponential $\lambda=1$ & $37.3 \%$ & $38.4 \%$ & $39.3 \%$ & $40.1 \%$ \\
\hline Normal $\mu=100, \sigma=30$ & $19.1 \%$ & $21.6 \%$ & $23.2 \%$ & $23.6 \%$ \\
\hline Normal $\mu=100, \sigma=50$ & $26.9 \%$ & $25.3 \%$ & $26.8 \%$ & $27.4 \%$ \\
\hline Pareto $\beta=2$ & $36.6 \%$ & $39.1 \%$ & $39.6 \%$ & $41.2 \%$ \\
\hline Pareto $\beta=1.1$ & $45.4 \%$ & $44.9 \%$ & $45.1 \%$ & $45.4 \%$ \\
\hline
\end{tabular}

Figure 6: Share of supply chain profit going to the buyer, for several demand distributions, and $c=0.2$.

each period, the supplier sets a price, and the buyer places an order for that price. The model is a direct extension to Lariviere and Porteus [10] from single-period to multi-period horizon.

We use the concept of sub-game perfection to define the optimal pricing (for the supplier) and purchasing (for the buyer) strategy, given the other player's actions. We find that, under some demand regularity conditions, including uniform, approximate normal and exponential demand, both the supplier's and the buyer's profit maximization problems are well-behaved, which is new to the literature.

In addition, we show that supply chain efficiency increases with the length of the negotiation $T$. Specifically, we show that the sub-optimality gap between the $T$-periods negotiation and the centralized supply chain falls with $1 / T$, regardless of the demand distribution. Thus, for large $T$, the negotiation situation approaches the highest possible efficiency for the supply chain.

Interestingly, our iterative approach provides an asymptotic coordination mechanism with a single profit sharing between buyer and supplier. Usually, coordination contracts, such as buy-back, have a degree of freedom that allows any profit sharing to occur. That is, buy-back allows for flexibility in the shares of profit gained by buyer and supplier. However, this also complicates the design of the contract, since the degree of freedom can lead to disagreement between players. In contrast, our proposed mechanism is more rigid, and, as a consequence, simpler. It provides a natural sharing of profits in the supply chain.

Finally, the paper provides some directions to design the negotiation process between the buyer and the seller. If negotiating is costly, how long the negotiation should last? This question can be answered using the asymptotic expected supply chain profit, from Theorem 4 ,

$$
S C_{T} \approx S C^{*}-\frac{f\left(z^{*}\right)\left(z^{*}\right)^{2}}{2 \Pi} \frac{1}{T}
$$

If conducting one additional negotiation round $\operatorname{costs} \kappa$, then the (approximate) optimal length 
of the negotiation should maximize

$$
S C^{*}-\frac{f\left(z^{*}\right)\left(z^{*}\right)^{2}}{2 \Pi} \frac{1}{T}-\kappa T
$$

and thus

$$
T^{*}=\sqrt{\frac{f\left(z^{*}\right)\left(z^{*}\right)^{2}}{2 \Pi \kappa}}
$$

Hence, the optimal negotiation length scales up with $\frac{1}{\sqrt{\kappa}}$. Hence, if negotiation costs could be cut by a factor 4 , then the negotiation length should be doubled. Thus, our basic model could potentially be used to study buyer-supplier interactions.

Furthermore, our work presents a number of interesting questions to be explored in the future.

First, our work focuses on the negotiation between one supplier and one buyer, both strategic. The revenue management literature has studied in a different setting the pricing problem of one supplier pricing against one buyer with probabilistic willingness-to-pay. Since the supplier maximizes its expected profit, this is equivalent to pricing against infinite buyers. This situation has been studied both for myopic buyers, see Lazear [11], and for strategic customers, see Besanko and Wilson [2]. Thus, both the one-buyer situation and the infinite-buyer situation have been studied. The $n$-buyers situation is the immediate extension of this work.

Second, following Granot et al. [9], the extension to the case of multiple suppliers is also interesting. In that situation, the buyer faces the trade-off between placing orders in the beginning, at a higher price, so that suppliers can offer lower prices, or wait for the suppliers to compete and reduce prices. This new trade-off may change the suppliers' behavior, compared to our model.

\section{References}

[1] Barnes-Schuster D., Y. Bassok and R. Anupindi 2002. "Coordination and Flexibility in Supply Contracts with Options." Manufacturing \& Service Operations Management, 4(3), pp.171-207.

[2] Besanko D. and W. Winston 1990. "Optimal Price Skimming by a Monopolist Facing Rational Consumers." Management Science, 36(5), pp. 555-567.

[3] Cachon G. P. 2003. "Supply Coordination with Contracts." Handbooks in Operations Research and Management Science: Supply Chain Management, edited by Steve Graves and Ton de Kok and published by North-Holland. 
[4] Cachon G. P. and M. A. Lariviere 2005. "Supply Chain Coordination with RevenueSharing Contracts: Strengths and Limitations." Management Science, 51(1), pp. 30-44.

[5] Debo L. G. and J. Sun 2004. "Repeatedly Selling to the Newsvendor in Fluctuating Markets: The Impact of the Discount Factor on Supply Chain Coordination." Working paper, Tepper School of Business, Carnegie Mellon University.

[6] Elmaghraby W. J. and P. Keskinocak 2003. "Dynamic Pricing in the Presence of Inventory Considerations: Research Overview, Current Practices, and Future Directions." Management Science, 49(10), pp. 12871309.

[7] Eppen G. and A. Iyer 1997. "Backup Agreements in Fashion Buying - The Value of Upstream Flexibility." Management Science, 43(11), pp. 1469-1484.

[8] Erhun F., P. Keskinocak and S. Tayur 2000. "Dynamic Procurement, Quantity Discounts, and Supply Chain Efficiency." Working paper, Department of Management Science and Engineering, Stanford University.

[9] Granot D., F. Granot and B. Mantin 2005. "A Dynamic Pricing Model under Duopoly Competition." Working paper, Sauder School of Business, University of British Columbia.

[10] Lariviere M. A. and E. L. Porteus 2001. "Selling to the Newsvendor: An Analysis of Price-Only Contracts." Manufacturing \& Service Operations Management, 3(4), pp. 293305.

[11] Lazear E. P. 1986. "Retail Pricing and Clearance Sales." American Economic Review, 76(1), pp. 14-32.

[12] Martínez-de-Albéniz V. and D. Simchi-Levi 2005. "A Portfolio Approach to Procurement Contracts." Production and Operations Management, 14(1), pp. 90-114.

[13] Pasternack B. A. 1985. "Optimal Pricing and Returns Policies for Perishable Commodities." Marketing Science, 4(2), pp. 166-176.

[14] Perakis G. and G. Roels 2005. "The Price of Anarchy in Supply Chains: Quantifying the Efficiency of Price-Only Contracts." Working paper, Operations Research Center, MIT.

[15] Rivero E. 2000. "Hollywood Entertainment, Rentrak settle 200 million lawsuit." Video Business, January 31st, 2000.

[16] Talluri K. and G. J. van Ryzin 2005. The Theory and Practice of Revenue Management. Springer Science+Business Media, New York, New York. 


\section{Proof of Theorem 1}

Proof. We prove the theorem for each $t$. First (buyer), as mentioned before the theorem, the buyer's problem is well-behaved when $u_{t}$ is decreasing.

Second (supplier), the final order quantity $x_{0}$ preferred by the supplier is unique and interior when

$$
s_{t-1}^{\prime}\left(x_{0}\right)+b_{t-1}^{\prime}\left(x_{0}\right)-c\left(1-\phi_{t-1}^{\prime}\left(x_{0}\right)\right)+u_{t}^{\prime}\left(x_{0}\right)\left(x_{0}-\phi_{t-1}\left(x_{0}\right)-x_{t}\right)=0
$$

has a unique solution; and the left-hand side is positive before, and negative after that solution.

Noting that $b_{t}\left(x_{0}\right)+s_{t}\left(x_{0}\right)=B_{0}\left(x_{0}\right)-c\left(x_{0}-x_{t}\right)=\int_{0}^{x_{0}} \bar{F}-c \phi_{t}\left(x_{0}\right)$, we have that $b_{t}^{\prime}\left(x_{0}\right)+$ $s_{t}^{\prime}\left(x_{0}\right)=\bar{F}\left(x_{0}\right)-c \phi_{t}^{\prime}\left(x_{0}\right)$, and Equation (16) can be rewritten as

$$
\bar{F}\left(x_{0}\right)-c+u_{t}^{\prime}\left(x_{0}\right)\left(x_{0}-\phi_{t-1}\left(x_{0}\right)-x_{t}\right)=0
$$

Hence, it is sufficient that

$$
\frac{\bar{F}\left(x_{0}\right)-c}{-u_{t}^{\prime}\left(x_{0}\right)}+\phi_{t-1}\left(x_{0}\right)
$$

does not increase more than $x_{0}$, i.e., its slope is no larger than 1 . In that case, we have

$$
x_{0}-x_{t}=\phi_{t-1}\left(x_{0}\right)+\frac{\bar{F}\left(x_{0}\right)-c}{-u_{t}^{\prime}\left(x_{0}\right)}=\phi_{t}\left(x_{0}\right) .
$$

This explains Equation (8).

Finally, we can rewrite the buyer's profit, i.e., $b_{t}\left(x_{0}\right)$ :

$$
b_{t}\left(x_{0}\right)=-u_{t}\left(x_{0}\right)\left(\phi_{t}\left(x_{0}\right)-\phi_{t-1}\left(x_{0}\right)\right)+b_{t-1}\left(x_{0}\right)
$$

which implies

$$
\begin{aligned}
b_{t}^{\prime}\left(x_{0}\right) & =b_{t-1}^{\prime}\left(x_{0}\right)-u_{t}\left(x_{0}\right)\left(\phi_{t}^{\prime}\left(x_{0}\right)-\phi_{t-1}^{\prime}\left(x_{0}\right)\right)-u_{t}^{\prime}\left(x_{0}\right)\left(\phi_{t}\left(x_{0}\right)-\phi_{t-1}\left(x_{0}\right)\right) \\
& =u_{t}\left(x_{0}\right)\left(1-\phi_{t}^{\prime}\left(x_{0}\right)\right)-u_{t}^{\prime}\left(x_{0}\right)\left(\frac{\bar{F}\left(x_{0}\right)-c}{-u_{t}^{\prime}\left(x_{0}\right)}\right)
\end{aligned}
$$

or equivalently

$$
u_{t+1}\left(x_{0}\right)=u_{t}\left(x_{0}\right)+\frac{\bar{F}\left(x_{0}\right)-c}{1-\phi_{t}^{\prime}\left(x_{0}\right)},
$$

Equation (9). The recursion given by Equation (17) completes the theorem. Note that the recursion (and hence the proof) is well-behaved if $\phi_{t}(x)-x$ and $u_{t}(x)$ are decreasing, the required condition. 


\section{Proof of Theorem 2}

Proof. This simply involves the change of variables $p=\bar{F}(x)-c$. Thus $\lambda_{t}(p)=\phi_{t}\left(\bar{F}^{-1}(p+c)\right)$ and $v_{t}(p)=u_{t}\left(\bar{F}^{-1}(p+c)\right)$ satisfy the recursion stated in the lemma, since

$$
\lambda_{t}^{\prime}(p)=\phi_{t}^{\prime}\left(\bar{F}^{-1}(p+c)\right) \cdot\left(-\frac{1}{f\left(\bar{F}^{-1}(p+c)\right)}\right)
$$

and

$$
v_{t}^{\prime}(p)=u_{t}^{\prime}\left(\bar{F}^{-1}(p+c)\right) \cdot\left(-\frac{1}{f\left(\bar{F}^{-1}(p+c)\right)}\right) .
$$

\section{Proof of Lemma 1}

Proof. We can verify easily that the recursion given by Equations (10) and (11) is satisfied by $\lambda_{t}=\bar{\lambda}_{t} p^{1-b}$ and $v_{t}=\bar{v}_{t} p+c$, where $\bar{\lambda}_{0}=0, \bar{v}_{1}=1$ and for $t \geq 1$,

$$
\bar{\lambda}_{t}=\bar{\lambda}_{t-1}+\frac{1}{a \bar{v}_{t}}, \quad \bar{v}_{t+1}=\bar{v}_{t}+\frac{1}{1+a(1-b) \bar{\lambda}_{t}} .
$$

The coefficients $\bar{\lambda}_{t}$ and $\bar{v}_{t}$ can be found observing that

$$
\begin{aligned}
\left((1-b) \bar{\lambda}_{t}+\frac{1}{a}\right) \bar{v}_{t+1} & =\left((1-b) \bar{\lambda}_{t}+\frac{1}{a}\right) \bar{v}_{t}+\frac{1}{a} \\
& =\left((1-b) \bar{\lambda}_{t-1}+\frac{1}{a}\right) \bar{v}_{t}+\frac{2-b}{a}
\end{aligned}
$$

Thus, using the initial conditions at $t=0$, we have that $\left((1-b) \bar{\lambda}_{t}+\frac{1}{a}\right) \bar{v}_{t+1}=\frac{(2-b) t+1}{a}$ and hence

$$
\bar{v}_{t+1}=\frac{(2-b) t+1}{a(1-b) \bar{\lambda}_{t}+1} .
$$

In addition, substituting this in the recursion for $\bar{\lambda}_{t}$ yields

$$
\bar{\lambda}_{t}=\bar{\lambda}_{t-1}+\frac{(1-b) \bar{\lambda}_{t-1}+\frac{1}{a}}{(2-b) t-1+b} .
$$

Thus,

$$
(1-b) \bar{\lambda}_{t}+\frac{1}{a}=\left((1-b) \bar{\lambda}_{t-1}+\frac{1}{a}\right)\left(1+\frac{1-b}{(2-b) t-1+b}\right)=\frac{1}{a} \prod_{k=1}^{t}\left(\frac{(2-b) k}{(2-b) k-1+b}\right)
$$


which implies

$$
\bar{\lambda}_{t}=\frac{1}{a(1-b)}\left\{\frac{\prod_{k=1}^{t}(2-b) k}{\prod_{k=1}^{t}(2-b) k-1+b}-1\right\}=\frac{1}{a(1-b)}\left\{\frac{\prod_{k=1}^{t}(2-b) k}{\prod_{k=1}^{t-1}(2-b) k+1}-1\right\} .
$$

Substituting this expression in Equation (18) yields

$$
\bar{v}_{t+1}=\{(2-b) t+1\}\left\{\frac{\prod_{k=1}^{t-1}(2-b) k+1}{\prod_{k=1}^{t}(2-b) k}\right\}=\frac{\prod_{k=1}^{t}(2-b) k+1}{\prod_{k=1}^{t}(2-b) k} .
$$

\section{Proof of Lemma 2}

Proof. The recursion around $p=0$ yields

$$
\begin{aligned}
& \lambda_{t}(0)=\lambda_{t-1}(0)+0 \\
& \lambda_{t}^{\prime}(0)=\lambda_{t-1}^{\prime}(0)+\frac{1}{g(0) v_{t}^{\prime}(0)}+0 \\
& v_{t+1}(0)=v_{t}(0)+0 \\
& v_{t+1}^{\prime}(0)=v_{t}^{\prime}(0)+\frac{1}{1+g(0) \lambda_{t}^{\prime}(0)}+0
\end{aligned}
$$

which results on the recursion used in Lemma 1 with $b=0$, i.e., $\lambda_{t}^{\prime}(0)=\bar{\lambda}_{t}$ and $v_{t}^{\prime}(0)=\bar{v}_{t}$.

\section{Proof of Lemma 3}

Proof. We prove it by induction. It is true for $t=0$. If it is true for $t-1$, at time $t$, we have:

$$
v_{t}^{\prime}=t\left(1-\frac{p}{r}\right)^{t-1} \text {. }
$$

Hence,

$$
\lambda_{t}=\left(\frac{1}{a}\right)\left\{\sum_{k=1}^{t-1} \frac{1}{k}\left(1-\frac{p}{r}\right)^{-k}\right\}+\frac{1}{a\left(1-\frac{p}{r}\right) t\left(1-\frac{p}{r}\right)^{t-1}}=\left(\frac{1}{a}\right)\left\{\sum_{k=1}^{t} \frac{1}{k}\left(1-\frac{p}{r}\right)^{-k}\right\}
$$


which yields

$$
\begin{aligned}
\lambda_{t}^{\prime} & =\left(\frac{1}{a r}\right)\left\{\sum_{k=1}^{t}\left(1-\frac{p}{r}\right)^{-k-1}\right\}=\left(\frac{1}{a r}\right)\left(1-\frac{p}{r}\right)^{-2}\left\{\frac{\left(1-\frac{p}{r}\right)^{-t}-1}{\left(1-\frac{p}{r}\right)^{-1}-1}\right\} \\
& =\left(\frac{1}{a p}\right)\left(1-\frac{p}{r}\right)^{-1}\left\{\left(1-\frac{p}{r}\right)^{-t}-1\right\} .
\end{aligned}
$$

Thus,

$$
\begin{aligned}
v_{t+1} & =c+r\left\{1-\left(1-\frac{p}{r}\right)^{t}\right\}+\frac{p}{1+a p\left(1-\frac{p}{r}\right) \lambda_{t}^{\prime}} \\
& =c+r\left\{1-\left(1-\frac{p}{r}\right)^{t}\right\}+\frac{p}{1+\left\{\left(1-\frac{p}{r}\right)^{-t}-1\right\}} \\
& =c+r\left\{1-\left(1-\frac{p}{r}\right)^{t}\right\}+r\left(\frac{p}{r}-1+1\right)\left(1-\frac{p}{r}\right)^{t} \\
& =c+r\left\{1-\left(1-\frac{p}{r}\right)^{t+1}\right\} .
\end{aligned}
$$

\section{Proof of Theorem 3}

Proof. From Theorem 1, we have that $\phi_{t}$ and $u_{t}$ are increasing in $t$. Thus, the solution to $\phi_{T}(x)=x$, that characterizes $x_{0}$ after $T$ negotiation rounds, is increasing in $T$. As a result, $S C_{T}=b_{T}\left(x_{0}\right)+s_{T}\left(x_{0}\right)=\int_{0}^{x_{0}} \bar{F}-c x_{0}$ also increases in $T$.

Finally, as a function of $T, x_{0}$ increases but cannot grow larger than $z^{*}$, since $p_{T}>c$ always. As a result, it converges to a finite limit. This limit $\bar{z}$ can be calculated from the recursion: it satisfies Equation (8) taken for large $t$, where $\phi_{t}(\bar{z})=\phi_{t-1}(\bar{z})=\bar{z}$ :

$$
\bar{z}=\bar{z}+\frac{\bar{F}(\bar{z})-c}{-u_{t}^{\prime}(\bar{z})},
$$

which can only hold when $\bar{F}(\bar{z})-c=0$, i.e., $\bar{z}=z^{*}$.

\section{Proof of Theorem 4}

Proof. Assuming that $f$ is sufficiently smooth around $z^{*}$, e.g., when it is infinitely differentiable near $z^{*}$, the Taylor expansion of $\lambda_{T}$ around $p=0$, using Lemma 2, is

$$
\lambda_{T}(p)=\frac{1}{f\left(z^{*}\right)}\left(\frac{2^{2 T}(T !)^{2}}{(2 T) !}-1\right) p+\epsilon^{\lambda}(p)
$$


where $\epsilon^{i}$ denote functions such that $\epsilon^{i}(t) / t \rightarrow 0$ when $t \rightarrow 0$. Using the reverse transformation of Theorem 2, around $z=z^{*}$, we have

$$
\phi_{T}(z)=\left(\frac{2^{2 T}(T !)^{2}}{(2 T) !}-1\right)\left(z^{*}-z\right)+\epsilon^{\phi}\left(z^{*}-z\right) .
$$

As a result, the solution to $\phi_{T}\left(z_{T}\right)=z_{T}$ can be also approximated, so that when $T \rightarrow \infty$ :

$$
\frac{z^{*}-z_{T}}{z *}\left(\frac{(2 T) !}{2^{2 T}(T !)^{2}}\right)^{-1} \rightarrow 1
$$

In addition, using the Stirling approximation, we have that

$$
\left(\frac{(2 T) !}{2^{2 T}(T !)^{2}}\right) \sqrt{\Pi T} \rightarrow 1
$$

This yields the result for $z^{*}-z_{T}$. The approximation of $S C^{*}-S C_{T}$ follows from

$$
S C^{*}-S C_{T}=\int_{z_{T}}^{z^{*}}\left(t-z_{T}\right) f(t) d t=\frac{f\left(z^{*}\right)\left(z^{*}-z_{T}\right)^{2}}{2}+\epsilon^{S C}\left(\left(z^{*}-z_{T}\right)^{2}\right) .
$$

